\title{
Gowers' syringal haemorrhage
}

\author{
C. B. SEDZIMIR, J. R. ROBERTS, J. V. OCCLESHAW, AND P. H. BUXTON
}

From the Regional Department of Medical and Surgical Neurology, Walton Hospital, Liverpool

SYNOPSIS The second case of Gowers' syringal haemorrhage, successfully treated by surgery, and the first such case in a child, is described. It is suggested that the very slow development of haematomyelia within an existing hydrosyringomyelia may originate from a torn intraspinal vein, deprived of its normal neural and glial support.

Perot et al. (1966) reviewed the history of the condition described by Gowers in 1904 and named Gowers' syringal haemorrhage by Wilson (1954-55). The first was the only one of Gowers' four postulated cases which was proven by necropsy. The second was the case of Perot et al. (1966) which was demonstrated by myelography and surgery.

The condition is that of a slow haemorrhage into an existing hydrosyringomyelia. Trauma was apparently not responsible for the haemorrhage in Gowers' patient. The patient described by Perot et al. was alleged to have had a fall six days before the onset of the first symptom which was pain in the neck and right shoulder. This was followed by numbness and paralysis of the right arm. The influence of the trauma upon the subsequent events was somewhat problematical but the authors stated that the haemorrhage was the result of that trauma. This patient was known to have had hydromyelia of the cervical cord in 1958 when myelography was performed for a lumbar intervertebral disc lesion. Thus the patient had had the hydromyelic condition for at least six years before the occurrence of the haemorrhage into it.

Our child appears to be only the second example of this condition verified and treated surgically. Trauma did not appear to play any part in its pathogenesis.

\section{CASE REPORT}

(Case no: 27589/69) A.J.L. was born on 16 August 1958. She was admitted in March 1969. There was no relevant family or personal history. She was born at full term; the delivery was uneventful. She was right handed.

Her first complaint was of pain at the root of the neck in February 1967, lasting only some hours. There was no injury and no special exertion to account for it. A similar attack occurred in September 1968 for about two days. The last attack commenced insidiously three weeks before the date of her examination.

For at least three months previously, she had noticed a gradual onset of clumsiness and weakness of the left hand and fingers. This became very marked and for the last four weeks she described that hand as being 'useless'. In fact, the last attack of pain only brought to attention the existing advanced weakness? of the left hand.

On examination she was found to be intelligent and cooperative and a better witness than her mother and father.

There was a left-sided mild hemi-atrophy of the face and of the left limbs and a left Horner's syndrome. There was a horizontal nystagmus on deviation of the eyes to the right. No other abnormalities were found in the cranial nerves. The right upper limb was normal. There was generalized weakness of the left shoulder, elbow, and wrist as well as gross weakness of the finger movements. All muscles of the left upper limb showed generalized wasting which was mild except for the gross atrophy of the thenar, interosseous, and hypothenar groups of muscles. Sensation to pin prick and temperature was depressed in the sixth cervical to second dorsal dermatomes on the left. The trunk showed a mild cervicodorsal kyphoscoliosis and loss of abdominal reflexes on the left.

The tone and power of the lower limbs were symmetrical and normal, although the left leg was thinner and the foot was smaller. She walked on a wide base and the heel-knee test was very clumsy 312 
bilaterally. All modalities of sensation in the lower limbs were normal, including joint position sense and vibration sense. The tendon reflexes were very brisk in all limbs but the plantar reflexes were flexor.

RADIOLOGICAL INVESTIGATIONS There was considerable widening of the cervical spinal canal and the pedicles of the upper four dorsal vertebrae appeared thinned.

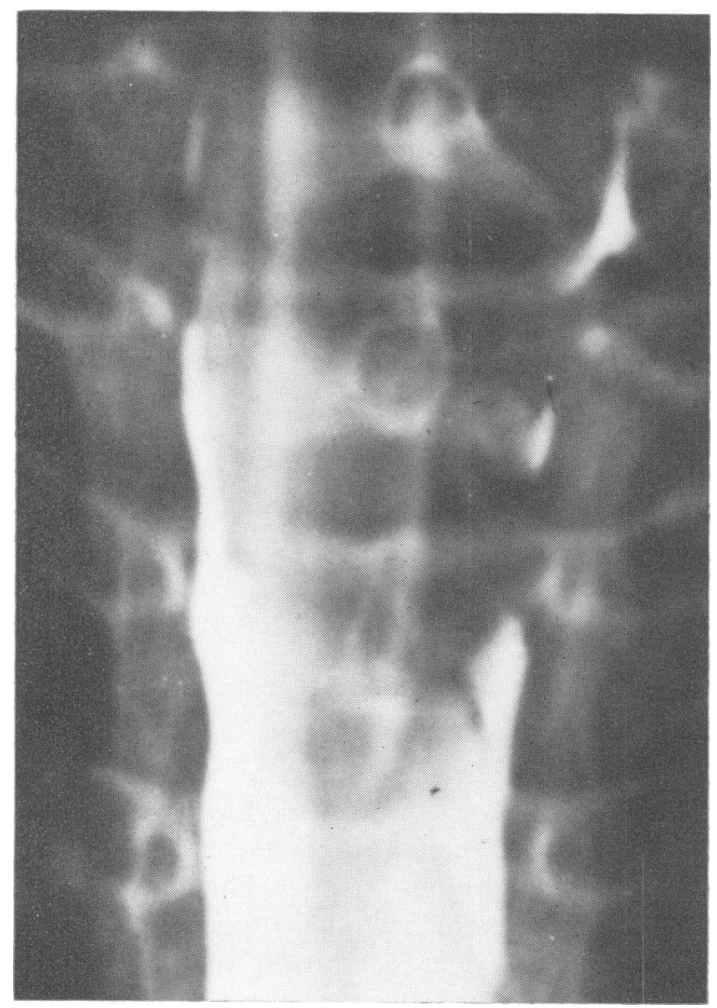

FIG. 1. Lumbar myelogram: an intramedullary lesion from the level of the third dorsal vertebra.

LUMBAR MYELOGRAPHY The contrast medium ran freely up to the level of D 4 vertebra where it was arrested in a fashion suggesting an intramedullary lesion (Fig. 1).

The cerebrospinal fluid contained $360 \mathrm{mg}$ protein per $100 \mathrm{ml}$. and 1 cell per c.mm.

Full blood examination, including erythrocyte sedimentation rate, was normal.

An electroencephalogram was also normal.

DIAGNOSIS The possibility of a slow haemorrhage into an existing hydrosyringomyelia or, alternatively, of a slowly growing cystic intramedullary tumour were considered. Positive contrast medium has been described to enter a hydrosyringomyelia from the 4th ventricle, therefore the following investigation was performed:

POSITIVE CONTRAST VENTRICULOGRAPHY In the sitting position, a right frontal burr hole was made and a radio-opaque catheter was inserted into the 3rd ventricle through the foramen of Monro, according to the technique described by Sedzimir and Iwan (1962). Using the image intensifier and closed circuit television, the radio-opaque medium was followed through the aqueduct and the 4th ventricle from which it escaped into the cisterna magna. Having established the patency of the foramen of Magendie, the medium was shaken down to the cervical canal where it showed a complete block at the level of C 4 vertebra. Regrettably, but understandably (see operation notes) the central cavity of the cervical spine was not demonstrated.

The ventricular cerebrospinal fluid contained 1 cell per c.mm and $32 \mathrm{mg}$ protein per $100 \mathrm{ml}$.

OPERATION A laminectomy of C 2 to D 3 vertebrae was performed. A tense spinal cord containing an intramedullary bluish mass was seen practically throughout the exposure (Fig. 2). The cord was firmly bound in arachnoidal adhesions and fixed tightly to the dura mater. These adhesions were divided, including the denticulate ligaments. The cerebrospinal fluid below the block was xanthochromic. Using a 22 gauge lumbar needle, about $18 \mathrm{ml}$. brownish, dark, decomposed fluid blood was aspirated and the cord 'collapsed' instantly.

A small incision was made on the left, between two denticulate ligaments; some more solid blood clot was aspirated without producing any fresh bleeding. A biopsy sample of the wall of the spinal cavity was taken. The spinal cord became completely slack and pulsatile (Fig. 3).

PATHOLOGICAL REPORT (20 March 1969) Only altered blood and no neoplastic or other cells were identified. Smear and paraffin sections of the tissue showed amorphous, sparsely cellular material. There were no tumour cells. Fragments of cord tissue showing degenerative neurones with marked homogenizing change and some increase in fibrillary astrocytes were present but no abnormal blood vessels were seen.

EXAMINATION: JUNE 1972 There was still atrophy of the left interosseous, thenar, and hypothenar muscles but the motor power had recovered to grade III. All other muscles were now grade IV to V. 


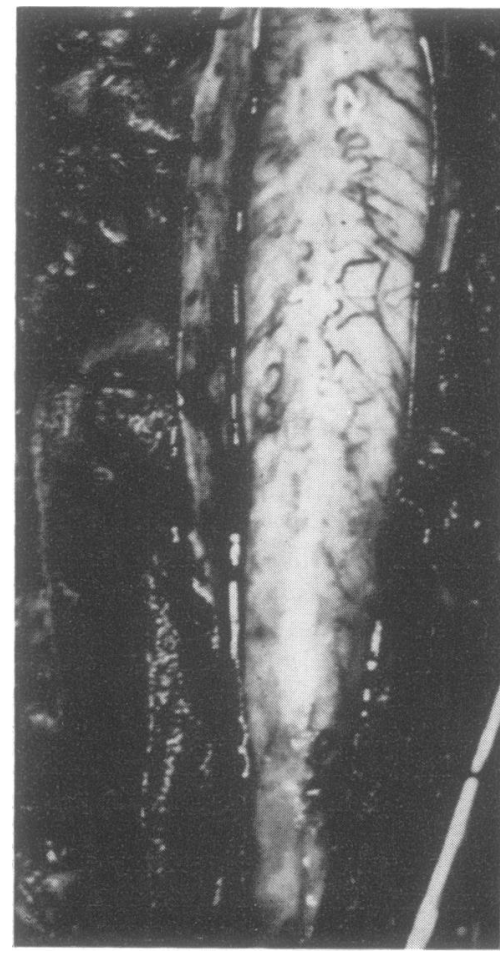

FIG. 2. (left) Intramedullary

haematoma in a syringomyelitic cavity, $C 3$ to $D 3$.

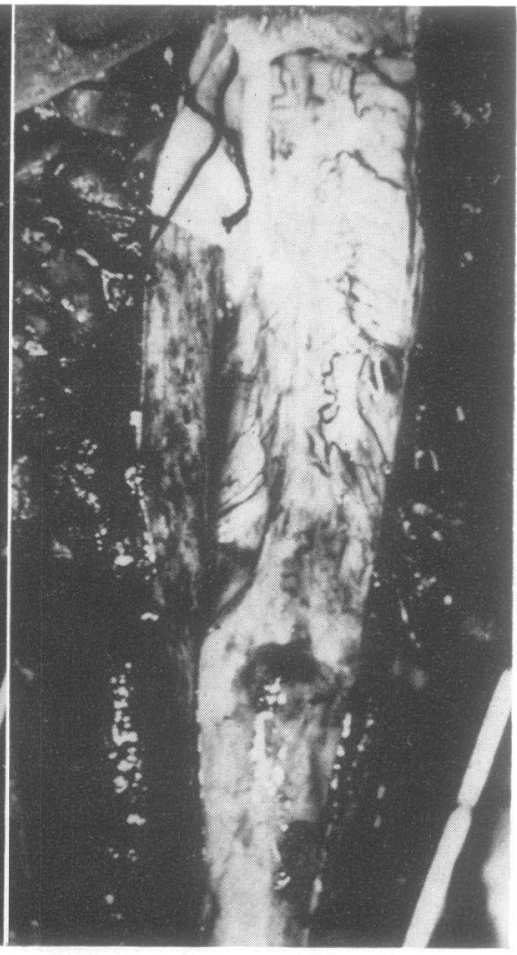

FIG. 3. (right) Same operative field after aspiration of the intramedullary haematoma.
The tendon reflexes were symmetrically exaggerated with bilaterally positive Hoffman's reflexes, but the plantar responses were flexor. Abdominal reflexes were absent. All modalities of sensation were normal throughout.

Despite some residual ataxia on knee-heel test, her gait was normal and she was riding a bicycle. She was always fully continent.

\section{DISCUSSION}

Cavitation of the cord is known to be the ultimate result of some traumatic, neoplastic, or haemorrhagic incidents-for instance, from an arteriovenous malformation (Odom et al. 1956; Poser, 1956; Rossier et al., 1968; and Williams and Turner, 1971).

By contrast with the very slow progress of the neurological disability and the even slower haemorrhage in our little girl, the development of the haematomyelia secondary to arteriovenous malformations or tumour is invariably dramatic. We could not elicit any evidence of injury, or even of an obvious strain. The history and progress of events would suggest a gradual hydrosyringomyelic cavitation of the cervicodorsal spinal cord for perhaps two years. Her parents were questioned repeatedly and thoroughly but were found to be extremely poor witnesses, while the girl was obviously trying to minimize her symptoms and signs.

Even during the last attack of cervical pain three weeks before her admission, there was no dramatic incident of neurological deterioration that would have suggested a sudden massive haemorrhage into the cord. Radiological evidence of long-standing widening of the cervicodorsal spinal canal was obvious.

We were not successful in demonstrating the entrance of radio-opaque medium into the 
cavity of the spinal cord but this was probably because of the blood clot filling the space.

The intramedullary haemorrhage into the hydrosyringomyelic cavity in our patient was entirely reminiscent of a history of a patient with a chronic intracranial subdural haematoma in the absence of any recognizable trauma. It is suggested that a slow venous haemorrhage into a relatively large space with subsequent liquification of the semi-solid clot into a fluid 'oily' decomposed blood took place. Osmotic mechanisms may have been instrumental here as they probably are in certain cases of chronic intracranial subdural haematomas.

How the haemorrhage begins is the question which is equally obscure in either situation, but probably more so in the present case. On one occasion, one of us (C. B. Sedzimir) had the opportunity of seeing the inside of what was a localized syringal cavity within the conus medullaris magnified $\times 6$ and illuminated by the Mark I Zeiss microscope (Sedzimir and Roberts, 1974). There was a venous blood vessel which looked normal, but floated in the fluid apparently unsupported. It may be that even a normal intraspinal vein may tear when it loses its support from surrounding glial and neural structures, producing a slow oozing.

In our child, the posterior columns were intact. Sensory changes were restricted to the crossing of the spinothalamic fibres at the cervical level. The corticospinal tracts were slightly and only temporarily compressed. The left cervical anterior horn cells were mainly affected, resulting in atrophy and weakness of the left hand and the Horner's syndrome.

\section{CONCLUSION}

Granted that our diagnosis is correct, then this child is an example of a very rare condition and, to our knowledge, she is only the second treated surgically with apparent success after a four years' follow-up.

\section{REFERENCES}

Gowers, W. R. (1904). Lecture 8. Syringal haemorrhage into the spinal cord. Lectures on Diseases of the Nervous System. 2nd series, pp. 200-220. Churchill: London.

Odom, G. L., Woodhall, B., and Margolis, G. (1957). Spontaneous hematomyelia and angiomas of the spinal cord. Journal of Neurosurgery, 14, 192-202.

Perot, P., Feindel, W., and Lloyd-Smith, D. (1966). Hematomyelia as a complication of syringomyelia: Gowers' syringal haemorrhage. Case report. Journal of Neurosurgery, 25, 447-451.

Poser, C. M. (1956). The Relationship between Syringomyelia and Neoplasm. Thomas: Springfield, Ill.

Rossier, A. B., Werner, A., Wildi, E., and Berney, J. (1968). Contribution to the study of late cervical syringomyelic syndromes after dorsal or lumbar traumatic paraplegia. Journal of Neurology, Neurosurgery, and Psychiatry, 31, 99-105.

Sedzimir, C. B., and Iwan, S. R. (1962). Simplified contrast ventriculography. Journal of Neurosurgery, 19, 657-660.

Sedzimir, C. B., and Roberts, J. R. (1974). Hydro-syringomyelia of the conus medullaris. Journal of Developmental Medicine and Child Neurology. (In press.)

Williams, B., and Turner, E. (1971). Communicating syringomyelia presenting immediately after trauma. Acta Neurochirurgica, 24, 97-106.

Wilson, S. A. K. (1954-55). Neurology. Edited by A. N. Bruce. 2nd edn. 3 vol. Butterworth: London. 